\title{
Clinical effectiveness of microsurgical subinguinal varicocelectomy with enhanced recovery after surgery for varicocele
}

\author{
Xiaobin Wang, Ruipeng Wang, Qiang Du, Bochen Pan \\ Center for Reproductive Medicine, Department of Obstetrics and Gynecology, Shengjing Hospital of China Medical University, Shenyang, China \\ Contributions: (I) Conception and design: B Pan; (II) Administrative support: B Pan; (III) Provision of study materials or patients: X Wang, Q Du; (IV) \\ Collection and assembly of data: R Wang; (V) Data analysis and interpretation: X Wang, R Wang; (VI) Manuscript writing: All authors; (VII) Final \\ approval of manuscript: All authors. \\ Correspondence to: Bochen Pan. Department of Gynecology, Center for Assisted Reproduction, Shenging Hospital of China Medical University, \\ Shenyang, China. Email: panbc@sj-hospital.org.
}

Background: Varicocele (VC) is one of the most common causes of infertility in men, and microscopic varicocelectomy is currently the major surgical procedure for $\mathrm{VC}$. We assessed the clinical effectiveness of microsurgical subinguinal varicocelectomy (MSV) with enhanced recovery after surgery (ERAS) in the treatment of $\mathrm{VC}$ in terms of semen quality improvement rate, pregnancy rate, pain relief rate, recurrence rate, and complication rate after MSV and explored the indications for VC surgery.

Methods: In total, 216 patients undergoing MSV in our center between June 2019 and July 2020 were enrolled in this study. All patients received the surgery under local anesthesia and were admitted and discharged within 24 hours. All patients were followed up for more than 6 months, and the rates of semen quality improvement, pregnancy, pain relief, recurrence, and postoperative complications were recorded. We can evaluate the pain degree of patients through the numerical rating scale (NRS). (I) 0 points for painless; (II) 1-3: mild pain; (III) 4-6: moderate pain; (IV) 7-10 points are severe pain. 0 is the most slightly, indicating comfort, and 10 is the most painful and unbearable.

Results: The sperm concentration, total sperm count, progressive motility rate, sperm viability, and morphology were significantly improved after the surgery (all $\mathrm{P}$ values $<0.05$ ). The rate of semen quality improvement was $88.2 \%$, and the semen indicators returned to normal in $26.6 \%$ of the patients. Among the patients who were followed up for 1 year, the natural conception rate reached $27.1 \%$ and was accompanied by a $95.5 \%$ pain relief rate, a $0.5 \% \mathrm{VC}$ recurrence rate, and a $2.3 \%$ postoperative complication rate. We obtained data through laboratory examination of semen DNA fragments index (DFI). Compared with preoperative and postoperative DFI, postoperative DFI was improved, and the pregnancy outcome was improved.

Conclusions: MSV under local anesthesia increases the rates of semen quality improvement, pregnancy, and pain relief while lowering the rates of recurrence and postoperative complications. MSV may also help to improve the pregnancy outcomes in patients with VC accompanied by sperm DNA fragmentation or nonobstructive azoospermia, but this should be verified by further investigation.

Keywords: Microsurgical subinguinal varicocelectomy (MSV); varicocele (VC); effectiveness; complication

Submitted Sep 02, 2021. Accepted for publication Oct 21, 2021.

doi: $10.21037 /$ tau-21-908

View this article at: https://dx.doi.org/10.21037/tau-21-908 


\section{Introduction}

Infertility is defined as the absence of a desired pregnancy, following regular, unprotected sexual intercourse for a period of at least 1 year (1). It affects an estimated $15 \%$ of couples globally, involving over 20 million men $(1,2)$. Males are found to contribute to about $50 \%$ of cases overall to various extent (1). There are many causes of male infertility, including erectile dysfunction, reproductive tract infections, endocrine disorders, immune infertility, genetic defects, and varicocele (VC). VC is defined as abnormal elongation, dilatation, and tortuosity of the veins in the pampiniform plexus of the spermatic cord, which can cause testicular pain, decreased testicular function, and even testicular atrophy. VC is considered to be one of the most common and correctable causes of male infertility. The prevalence of VC is about $10-15 \%$ in the general male population but can reach $30-40 \%$ in males with primary infertility.

The main causes of varicocele include two aspects: (I) congenital anatomical abnormalities, such as nutcracker syndrome, weak venous wall, poor venous valve function and so on. (II) Acquired factors, such as regular standing, sedentary, vigorous exercise, especially those activities that increase abdominal pressure and hinder spermatic venous reflux. (III) There are rare intra-abdominal tumors or other factors that compress the spermatic vein and lead to venous reflux.

There are two main indications for VC surgery in adults: (I) pain or cramps in the testicles and (II) abnormal semen parameters in infertile men. When VC causes testicular atrophy or decreased testicular function that affects growth and development, surgery may be a feasible treatment. Other indications for surgery include nonobstructive azoospermia, androgen deficiency, poor semen quality prior to assisted conception, and sperm fragmentation or sperm oxidative stress (3). Treatments of $\mathrm{VC}$ are divided into three main categories: (I) general therapy, including scrotal support and lifestyle modification; (II) pharmacological therapy, including administration of flavonoids (diosmin), sodium aescinate (Mai Zhi Ling), and traditional Chinese herbs for activating blood circulation and resolving blood stasis; and (III) surgical treatment, including microscopic surgery, laparoscopic surgery, interventional embolization, and open surgery (4). Among these, microsurgical varicocelectomy has gradually become the preferred surgical option for VC due to its advantages of less surgical damage, lower postoperative recurrence rate, fewer complications, higher postoperative semen quality, and higher postoperative fertility rate (4-6). The two approaches to microsurgical varicocelectomy include the inguinal or subinguinal routes. The subinguinal approach involves the shallow spermatic cord and simple anatomic layers; it does not require the dissection of the external abdominal oblique muscle and allows for the application of local anesthesia. Thus, it entails less surgical trauma and a shorter recovery time (7). However, it involves a large number of veins and thin testicular arteries, which accordingly increase intraoperative Doppler use and prolong the operating time under the microscope (8).

Microsurgical subinguinal varicocelectomy (MSV) with enhanced recovery after surgery (ERAS) has routinely been applied in our center. Here, we describe the surgical approach and report the follow-up results in terms of postoperative semen status, pregnancy rate of the spouse, pain relief rate, and postoperative complications.

Our work is completed under ERAS mode, and the anesthesia mode is completed under local anesthesia. This management mode and anesthesia method can greatly reduce the operation cost of patients, improve the medical experience of patients, and will not affect the operation effect. It is a relatively innovative management mode and anesthesia mode.

In our center, ERAS mode is mainly that patients arrive at the hospital after breakfast on the agreed operation date. After going through hospitalization procedures, operation signature and other procedures, they undergo spermatic vein ligation under the microscope for about 2 hours later. Patients can move on their own immediately after operation, and can leave the hospital and go home after a short observation for half an hour. Does not affect daily life. Instruct the patient not to exercise violently immediately. There is no need for special nursing, intravenous infusion, antibiotic treatment, incision and dressing change. Generally, it can work normally the next day.

Postoperative complications mainly included incision scar, postoperative testicular and incision pain, poor incision healing and delayed healing. There are no complications such as postoperative testicular ischemia atrophy and testicular hydrocele. We present the following article in accordance with the STROBE reporting checklist (available at https://dx.doi.org/10.21037/tau-21-908).

\section{Methods}

\section{Patients}

A total of 334 patients underwent MSV under local anesthesia 
Table 1 General information of the patients $(n=216)$

\begin{tabular}{|c|c|}
\hline General information & Values \\
\hline \multicolumn{2}{|l|}{ Age (years) } \\
\hline Mean \pm standard deviation & $31.0 \pm 4.6$ \\
\hline Range & $18-46$ \\
\hline \multicolumn{2}{|l|}{ Age of spouse (years) } \\
\hline Mean \pm standard deviation & $30.5 \pm 3.5$ \\
\hline Range & $20-43$ \\
\hline \multicolumn{2}{|l|}{ BMI $\left(\mathrm{kg} / \mathrm{m}^{2}\right)$} \\
\hline Mean \pm standard deviation & $25.3 \pm 3.8$ \\
\hline Range & $16.97-35.91$ \\
\hline \multicolumn{2}{|l|}{ Preoperative spermatic vein diameter $(\mathrm{mm})$} \\
\hline Left side & $2.9 \pm 0.6$ \\
\hline Right side & $2.5 \pm 0.6$ \\
\hline \multicolumn{2}{|l|}{ Testicular size (mL) } \\
\hline Left side & $14.8 \pm 4.0$ \\
\hline Right side & $15.2 \pm 3.9$ \\
\hline \multicolumn{2}{|l|}{ Surgical indications (n, \%) } \\
\hline Infertility combined with poor semen quality & $167(77.3)$ \\
\hline Miscarriage combined with poor semen quality & $11(5.1)$ \\
\hline Testicular pain only & $22(10.2)$ \\
\hline Azoospermia & $16(7.4)$ \\
\hline \multicolumn{2}{|l|}{ Dominant side of varicocele $(n, \%)$} \\
\hline Left side & $117(54.2)$ \\
\hline Both sides & $99(45.8)$ \\
\hline \multicolumn{2}{|l|}{ Varicocele grade $(\mathrm{n}, \%)$} \\
\hline Grade 1 & 0 \\
\hline Grade 2 & $96(44.4)$ \\
\hline Grade 3 & $120(55.6)$ \\
\hline
\end{tabular}

in our center between June 2019 and July 2020. Among them, there were 285 infertile patients and 49 patients with other conditions (including 16 patients with azoospermia, 11 patients whose spouses had a history of miscarriage, and 22 patients with simple testicular pain). Then, for our study, 167 patients were screened from the infertile patients, and the screening criteria are described in section 2.4. Thus, a total of 216 patients ( 167 infertile patients +49 patients with other conditions) were included in the final analysis.
The age of the patients and their spouses, body mass index (BMI), bilateral testicular size, surgical side, VC grade, and spermatic vein width were recorded (Table 1). All patients were followed up for more than 6 months, and the rates of semen quality improvement, pregnancy, pain relief, recurrence, and postoperative complications were recorded. The study was approved by Ethics Committee of Shengjing Hospital of China Medical University (No.2020PS083J). The study was conducted in accordance with the Declaration of Helsinki (as revised in 2013).

\section{Surgical algorithm}

Patients received preoperative examinations at the outpatient department first, at which time they received explanations concerning the characteristics of the surgery performed under local anesthesia and scheduled the date of surgery. On the day of admission, the patient could eat and drink normally, and the operation was performed after informed consent was obtained. Intraoperative neurophysiologic monitoring was performed during the surgery, and the nervousness and anxiety of the patients were alleviated by oral communication and playing music. After the operation, patients were observed with blood pressure and electrocardiogram (ECG) monitoring for 1 hour in the ward and were discharged upon the instruction of a senior physician. The discharge precautions were explained. All patients were discharged on the same day after surgery and were required to be accompanied by an adult for 24 hours.

\section{Surgical methods}

MSV was performed in all patients. Local anesthesia was delivered by the injection of $2 \%$ lidocaine $(20 \mathrm{~mL})$ and $1 \%$ ropivacaine $(10 \mathrm{~mL})$. No sedative, analgesic, or anxiolytic drugs were used before or after surgery, and no spinal anesthesia or intravenous anesthesia was applied during surgery.

For the procedure, the patient was placed in a supine position, and disinfection (with $0.5 \%$ iodophor) and draping were routinely performed. The left external inguinal ring was located near the pubic tubercle by palpation, and the incision site was marked below the annulus of the left external ring. The incision site was anesthetized by local infiltration into the surface of the spermatic cord. A $1.5-$ to $3-\mathrm{cm}$ oblique incision was made along the skin line. After the tissues were dissected layer by layer, the incision was exposed with a pull hook until 
the spermatic cord became visible. After the spermatic nerve was blocked, the spermatic cord was lifted out with appendage forceps and was followed by the placement of a rubber tube below the spermatic cord. The external spermatic vein was located and ligated. After the cremaster muscle was opened longitudinally at its thinnest site, the spermatic cord was separated into different parts (cremaster muscle, vas deferens, and internal spermatic cord fascia) along the gap between the cremaster muscle and vas deferens and that between the vas deferens and inner spermatic cord fascia. During the procedure, the testis was not delivered and the gubernacular veins were not handled. The microscopic operation was performed at $10 x$ to $20 \times$ magnification. The inner spermatic cord fascia was opened microscopically to locate the testicular arteries and lymphatic vessels, which were then protected by sutures. At least 1 artery and 3 lymphatic vessels were identified for each side during the operation. All veins were carefully searched for, skeletonized, mobilized, double ligated with 4-0 silk threads, and then transected. When there was no missed veins or bleeding spots visible to the naked eye on the same plane, the spermatic cord was returned to its usual position. The incision was closed layer by layer by with 3-0 absorbable sutures, and subcutaneous purse-string continuous sutures were used on the outer skin incision. Subsequently, the incision was protected with tissue glue and covered with a sterile dressing. The same method was used to treat the contralateral side.

\section{Screening criteria of patients}

All patients were clinically diagnosed with VC, and the inclusion and exclusion criteria are described below.

The inclusion criteria for the infertility group were the following: (I) failure to achieve pregnancy after 12 months or more of regular unprotected sexual intercourse and (II) presence of at least 1 abnormal parameter on preoperative semen examination.

The inclusion criterion for the pain group was intermittent or persistent sensation of swelling, hidden pain, or dull pain in the scrotal or groin area.

Inclusion criteria for the miscarriage group were the following: (I) a history of 1 or more spouse miscarriages and (II) the presence of at least one abnormal parameter on preoperative semen examination.

The inclusion criteria for the azoospermia group were the following: (I) no sperm found in more than two semen tests and (II) a clinical diagnosis of primary nonobstructive azoospermia.

The exclusion criteria included (I) refusal to cooperate during follow-up; (II) accompaniment of by retrograde ejaculation, erectile dysfunction, or ejaculation failure; (III) chromosomal abnormality or microdeletion in azoospermia factor (AZF) region; (IV) presence of hyperthyroidism, Cushing's syndrome, hypopituitarism, or pituitary tumor; (V) presence of severe malnutrition, severe diabetes mellitus, or other metabolic diseases; (VI) presence of prostatitis, seminal vesiculitis, urethritis, or leukocytospermia; (VII) presence of reproductive system malformation or absence of vas deferens; (VIII) a history of previous VC surgery or traction of cryptorchidy or experience of testicular atrophy after orchitis.

For patients included in the pregnancy rate statistics, the following exclusion criteria were also applied: (I) a history of spouse miscarriage within 1 year before surgery; (II) failure to have regular sexual intercourse due to contraception or other reasons after surgery; (III) a postoperative follow-up period shorter than 1 year; and (IV) abnormal reproductive function of the spouse, such as tubal blockage, ovulation disorder, or chromosomal abnormality, as assessed by reproductive gynecologists.

\section{Assessment indicators}

VC diagnosis was based on palpation and ultrasound findings. The relevant color Doppler ultrasound findings were the following: inner diameter of the spermatic veins larger than $2 \mathrm{~mm}$ in the calm state, significant increase in the inner diameter of the vein after increasing abdominal pressure, or was notable venous return after a Valsalva test. The VC was categorized based on the Dubin and Amela grading system as follows: grade I, varicose veins that can be palpated only after the Valsalva maneuver; grade II, varicose veins that can be palpated without the Valsalva maneuver but cannot be seen; and grade III, visible varicose veins. The grading was determined by palpation by two surgeons independently.

The testicular volume was determined by the Prader orchidometer or calculated with ultrasound measurements using the formula: testicular volume $=$ length $(\mathrm{L})(\mathrm{cm}) \times$ width $(W)(\mathrm{cm}) \times$ height $(\mathrm{H})(\mathrm{cm}) \times 0.71$.

Semen testing required the patient to be abstinent for 3-7 days (minimum 48 hours) and was based on the World Health Organization (WHO) Laboratory Manual for the Examination and Processing Human Semen (5th edition).

To determine the presence of recurrence, physical 
Table 2 Comparison of semen quality before and after surgery $(\mathrm{n}=169)$

\begin{tabular}{lcccc}
\hline Variable & Before surgery & After operation & t value & P value \\
\hline Semen $\mathrm{pH}$ & $7.39 \pm 0.05$ & $7.40 \pm 0.03$ & -1.09 & -0.96 \\
Semen volume $(\mathrm{mL})$ & $3.8 \pm 1.6$ & $3.9 \pm 1.5$ & -2.35 & 0.335 \\
Sperm concentration $\left(10^{6} / \mathrm{mL}\right)$ & $32.79 \pm 35.30$ & $38.81 \pm 33.00$ & -3.42 & 0.020 \\
Total sperm count $\left(10^{6}\right)$ & $115.48 \pm 106.23$ & $144.35 \pm 121.45$ & -5.65 & 0.001 \\
Forward rate $(\%)$ & $15.44 \pm 11.66$ & $20.58 \pm 14.12$ & -5.59 & 0.000 \\
Total motility rate $(\%)$ & $20.15 \pm 13.37$ & $25.96 \pm 16.85$ & $3.77 \pm 2.48$ & -3.05 \\
Rate of normal morphology $(\%)$ & $2.51 \pm 3.00$ & & 0.000 \\
\hline
\end{tabular}

Table 3 Summary of postoperative outcomes

\begin{tabular}{lc}
\hline Variable & Postoperative outcomes (n, \%) \\
\hline Spontaneous pregnancy within 1 year after surgery & $23 / 85(27.1)$ \\
Semen quality improvement rate & $149 / 169(88.2)$ \\
Total sperm count improvement rate & $116 / 169(68.6)$ \\
Forward progression improvement rate & $114 / 169(67.5)$ \\
Overall sperm motility improvement rate & $108 / 169(63.9)$ \\
Rate of sperm quality returned normal & $45 / 169(26.6)$ \\
Rate of testicular pain relief & $21 / 22(95.5)$ \\
Testicular pain only & $87 / 88(98.9)$ \\
Infertility combined with testicular pain & \\
\hline
\end{tabular}

examination and color Doppler ultrasound were reperformed 6 months after surgery, and recurrence was considered when the respective aforementioned criteria were met on both examinations.

\section{Statistical analysis}

Data were analyzed using SPSS 20.0 software package (IBM Corp., Armonk, NY, USA). Measurement data are presented using mean \pm standard deviation $($ mean $\pm \mathrm{SD}$ ) and were compared using the $t$-test, with a $\mathrm{P}$ value of less than .05 being considered statistically significant.

\section{Results}

A total of 169 patients underwent semen examination before and after surgery, and the sperm count, vitality, and morphology were significantly improved after surgery, with the differences for each being statistically significant
$(\mathrm{P}<0.05)$ (Table 2). The rate of semen quality improvement was $88.2 \%$, and the semen indicators returned normal in $26.6 \%$ of the patients. Among the 22 patients who underwent surgery due to testicular pain only, 1 case did not experience significant improvement after surgery, yielding an overall improvement rate of $95.5 \%$. The remaining 88 infertile patients also had testicular pain, and 1 case did not have significant pain relief after surgery, yielding an overall improvement rate of $98.8 \%$ (Table 3).

After the exclusion criteria were applied for the infertile patients, spousal pregnancies were analyzed for 85 patients, which revealed spontaneous pregnancies in 23 individuals $(27.1 \%)$, assisted pregnancies in 4 individuals $(4.7 \%)$, firsttrimester miscarriages in 2 individuals (2.4\%), ectopic pregnancy in 1 individual $(1.2 \%)$, and no pregnancy in 55 individuals $(64.7 \%)$. Detailed data on the time of pregnancy after surgery in 23 individuals are shown in Table 4.

Among the 16 azoospermia patients, a diagnosis of primary nonobstructive azoospermia was made as the 
Table 4 Patients who achieved a spontaneous conception $(\mathrm{n}=85)$

\begin{tabular}{lc}
\hline Variable & Value $(\mathrm{n}, \%)$ \\
\hline Spontaneous conception & \\
Within 3 months after surgery & $6(7.1)$ \\
Within 3-6 months after surgery & $5(5.9)$ \\
Within 7-9 months after surgery & $6(7.1)$ \\
Within 10-12 months after surgery & $6(7.1)$ \\
Total & $23(27.1)$ \\
\hline
\end{tabular}

Table 5 Postoperative complications ( $\mathrm{n}=216)$

\begin{tabular}{lc}
\hline Postoperative complications & Value $(\mathrm{n}, \%)$ \\
\hline Incision infection & 0 \\
Scrotal edema & $2(0.9)$ \\
Incisional fat liquefaction & $2(0.9)$ \\
Orchitis- and epididymitis & 0 \\
Testicular atrophy & 0 \\
Vas deferens injury & 0 \\
Postoperative testicular pain & $1(0.5)$ \\
Hydrocele & 0 \\
Total & $5(2.3)$ \\
\hline
\end{tabular}

possibilities of obstructive azoospermia and acquired factors (e.g., radiochemotherapy, postmumps orchitis, and cryptorchidism) were ruled out and the patients chromosomes and AZF were found to be normal. After the surgery, the presence of sperm in urinary sediment was detected in 1 patient after 4 months of conservative treatment; the patient was pathologically confirmed as having Sertoli cell-only syndrome and underwent intracytoplasmic sperm injection (ICSI). His wife had been pregnant for 6 months as of the follow-up date. The remaining 15 patients did not have sperm in their postoperative semen, among whom 4 patients did not obtain successful sperm retrieval under microscope, 5 chose donated sperm for conception, and 6 were treated with conservative medications. Of the 16 patients with biopsy results, 4 had primary spermatocyte maturation arrest, 2 had block of spermatogonial differentiation, and the remaining 10 had Sertoli cell-only syndrome. Of the 11 patients whose spouse had a history of miscarriage, the outcomes of their spouses included spontaneous pregnancies within 1 year after surgery ( $\mathrm{n}=2$, both delivered), recurrent miscarriages $(\mathrm{n}=2)$, and no pregnancy $(\mathrm{n}=7)$.

One patient $(0.5 \%)$ suffered from VC recurrence. The incidence of postoperative complications was $2.3 \%$, and included scrotal edema $(n=2)$, fat liquefaction $(n=2)$, and testicular pain after surgery $(n=1)$. No hydrocele, vas deferens injury, orchitis and epididymitis, or testicular atrophy were noted (Table 5). Fat liquefaction was improved after the wound dressing was changed at the outpatient office, scrotal edema was alleviated after oral administration of diosmin, and postoperative pain was insidious and managed with oral painkillers at the outpatient office.

\section{Discussion}

\section{$V C$ and male infertility}

Infertility has now become a major health disorder worldwide. Semen quality is directly related to pregnancy, miscarriage, and success of assisted reproductive techniques. Among these, $\mathrm{VC}$ is the most common cause of male infertility. The etiology of infertility caused by VC remains unknown, but the possible pathophysiological mechanisms may include oxidative stress, high scrotal temperature, hypoxia, regurgitation of renal and adrenal metabolites, hormonal imbalance, and production of antisperm antibodies $(3,9)$. Oxidative stress can directly or indirectly affect the spermatogenic cells and the basement membrane of the contorted seminiferous tubule, causing apoptosis. Higher scrotal temperature in VC patients affects testicular function. Local ischemic reaction may occur when venous pressure is higher than arterial pressure. The left spermatic vein joins the left renal vein, and venous return will cause the regurgitation of renal and adrenal metabolites, such as prostaglandins and epinephrine, leading to local vasoconstriction and jeopardizing spermatogenesis (9).

There are currently many surgical options for $\mathrm{VC}$, mainly open surgery, laparoscopic surgery, and microscopic surgery. A meta-analysis by Yuan et al. included seven clinical studies with 1,781 patients and found that microscopic surgery had the lowest incidence of postoperative complications and recurrence rates among these 3 surgical methods, but involved longer surgical time (10). Research has also shown that microscopic surgery is associated with the highest rates of semen quality improvement and conception $(4,11,12)$. A meta-analysis by Kim et al. in 2013 reported a conception rate of $21.8 \%$ after VC surgery as compared to $11.0 \%$ in the untreated group (13). In our current study, the semen 
quality improvement rate was $88.2 \%$, and the conception rate within 1 year after surgery was $27.1 \%$. The lower conception rate in this study may be explained by the short follow-up period. In addition, some patients showed no improvement or even a decrease in semen quality after surgery, and previous reports also noted that postoperative outcomes were not always improved $(14,15)$. In fact, there are many causes of male infertility, and $\mathrm{VC}$ is only one of them. Thus, other possible causes need to be strictly excluded before surgery.

About $2-10 \%$ of VC patients may suffer from pain, which manifests as intermittent or persistent cramping, or vague, dull pain in the scrotal or inguinal region and may be aggravated with prolonged standing. Resting in a supine position may help to relieve VC-related pain (16). The probable mechanisms for VC-related pain include elevated testicular temperature, increased venous pressure, hypoxia, oxidative stress, hormonal imbalances, and the reflux of toxic metabolites of adrenal or renal origin (17). In our study, the incidence of infertility with concomitant pain was $52.6 \%$, which may be related to the fact that $\mathrm{VC}$ was moderate or severe in all patients although psychological factors might also play a role. Pain can be relieved after surgery in more than $90 \%$ of VC patients (18). In 2016, a meta-analysis that included 12 studies indicated that the pain resolution rate was significantly higher after microsurgery than after laparoscopic varicocelectomy ( $\mathrm{RR}=0.77$; $95 \%$ CI: 0.60-0.99; $\mathrm{P}=0.04$ ), whereas the pain resolution rate was significantly higher after subinguinal varicocelectomy than after high or inguinal varicocelectomy $(\mathrm{RR}=0.83,95 \% \mathrm{CI}: 0.76-0.90$; $\mathrm{P}<.00001$ and $\mathrm{RR}=0.92 ; 95 \%$ CI: 0.86-0.99; $\mathrm{P}=0.02)(19)$. In our current study, the postoperative pain resolution rate was $95.5 \%$ for those who underwent MSV for testicular pain only, which was higher than the average pain resolution rate reported in the literature, which may be explained by the surgical procedure and the small sample size (3).

Compared with other surgical methods in the treatment of VC, it has obvious advantages in ERAS mode. (I) It can greatly reduce the length of hospital stay from a few days to a few hours. (II) it can greatly reduce the medical expenses of patients. (III) it can greatly improve the medical experience of patients, because there is no need for fasting water before operation, normal life before and after operation, and no need for intravenous infusion. (IV) It can greatly reduce the work intensity of medical staff without doing more work of little significance, such as night shift, such as recording disease records, such as intravenous infusion, etc. (IV) It can greatly increase the utilization rate of hospital beds and reduce operating costs.

\section{Complications}

Day surgery wards, as a new ward model that offers fast and convenient services for patients, can save medical resources, accelerate bed turnover, and also facilitate the efficient use of medical resources. Day surgery shortens the length of hospital stay, which lowers the incidence of hospital-acquired infections and reduces the disruption to patients' lives. However, short-term hospital stay may also lead to poor wound care if the postdischarge compliance is poor. In a Danish multicenter study of morbidity after 57,709 day surgery procedures, the overall rate of return hospital visits was $1.21 \%, 0.50 \%$ of which were due to hemorrhage or hematoma, $0.44 \%$ to infection, and $0.03 \%$ to thromboembolic events (20). In addition, preoperative and intraoperative anxiety is common among all patients undergoing local anesthesia. To address this problem, a detailed explanation of the procedure may be provided before surgery (21); during the surgery, playing music and talking with the patient may also be helpful. The combined use of sedative drugs and antianxiety drugs (such as diazepam) has also been reported (22).

Incisional fat liquefaction, a common postoperative complication, refers to the aseptic degeneration and death of fat cells, which results in overflow and aggregation of lipid droplets, manifest as redness, swelling, and pressure pain of the incision, along with visible yellow exudate. The main risk factors for fat liquefaction include obesity, improper suturing technique, use of a high-frequency electric knife, excessive intraoperative stretching of the incision, and diabetes [40]. In our current study, both patients $(0.9 \%)$ with fat liquefaction were obese patients $(\mathrm{BMI}>25)$. An electric knife was used during subcutaneous separation, and the adipose tissue was excessively stretched with a skin hook when the spermatic cord was being located, resulting in rupture of the fat cells. Treatment modalities for fat liquefaction include debridement and drainage, negative pressure wound therapy (NPWT), light irradiation therapy, and Chinese herbal therapy (23). The fat liquefaction in these two patients was improved after the wound dressing was changed and adequate drainage was performed at outpatient offices. For patients undergoing day surgery for $\mathrm{VC}$, especially those with obesity, intraoperative use of the electric knife in the fat layer should be reduced and full-thickness suturing should be achieved. In addition, discharge education should be conducted. Early detection, 
early diagnosis, and early treatment are essential for early recovery.

Scrotal edema and hydrocele are the most common complications after VC surgery. Al-Kandari et al. compared open, laparoscopic, and microscopic procedures for VC and found a scrotal edema incidence of $13 \%, 20 \%$, and $0 \%$, respectively (4). The testicular venous return system mainly includes the internal spermatic vein, the vas deferens vein, and the external spermatic venous system (including the cremasteric vein and gubernacular veins). The lymphatic vessels are translucent in appearance. When other veins are ligated, the lymphatic vessels are significantly thickened compensatorily to enhance the return. Lymphatic vessel injury may result in an increased incidence of hydrocele (24). In our study, we also ligated the external spermatic vein, cremasteric vein, and vas deferens vein (all of which have a diameter of $1 \mathrm{~mm}$ or more) during the surgical procedure, without handling the gubernacular veins (4). The gubernacular veins start at the caudal pole of the testis and are communicating branches between the deep veins (internal spermatic vein, cremasteric vein, and vas deferens vein) and the superficial veins (scrotal veins). Testicular delivery is needed if the gubernacular veins are ligated, which not only requires an enlarged incision (which increases the difficulty of the local anesthesia and the risk of testicular injury) but also may result in impaired venous return and scrotal edema, along with an increased incidence of hydrocele $(25,26)$. A meta-analysis in 2019 revealed that ligation of the gubernacular veins resulted in a higher incidence of orchiepididymitis ( $\mathrm{RR}=4.36 ; 95 \%$ CI: $1.12-16.99 ; \mathrm{P}=0.034)$ and scrotal oedema $(\mathrm{RR}=4.25 ; 95 \%$ CI: 2.40-7.54; $\mathrm{P}<0.001)$ and showed no beneficial influences on semen parameters or natural conception (27). In addition, whether the gubernacular veins were handled or not did not have any effect on the VC recurrence rate (28). In our study, no hydrocele occurred postoperatively, and the incidence of scrotal edema was only $0.9 \%$, which was associated with the choice of microscopic surgery, the lack of intraoperative handling of the gubernacular veins, and the intentional preservation of the lymphatic vessels.

$\mathrm{VC}$ recurrence is one of the most common complications after VC surgery. The reported VC recurrence rates were $14.97 \%, 4.3 \%, 1.05 \%$, and $12.7 \%$, respectively, after open surgery, laparoscopic surgery, microscopic surgery, and interventional embolization (9). The cause of recurrence is unclear. It may be due to unligated external spermatic veins after a microscopic high inguinal varicocelectomy or to the missed ligation of tiny veins during a microscopic subinguinal varicocelectomy (29). Microscopic surgery is routinely performed at up to $10 x-20 x$ magnification; even so, tiny nerves are still difficult to identify and separate. One of our patients had no testicular pain before surgery and suffered from persistent testicular pain during the follow-up, which might have been related to incomplete intraoperative venous skeletonization, which resulted in ligation of the fine nerves. Shiraishi et al. reported 3 patients with persistent scrotal pain after treatment with the subinguinal approach, which might be associated with the more complex course of the subinguinal nerve branches (8).

Testicular atrophy due to intraoperative arterial injury is one of the most serious complications of VC surgery. Chan et al. evaluated 2,102 cases of microsurgical varicocelectomy and found that the incidence of accidental testicular artery ligation in microsurgical varicocelectomy was $0.9 \%(\mathrm{n}=19)$; among those affected, 1 (5\%) developed testicular atrophy (30). Neither postoperative testicular atrophy nor vas deferens injury was seen in our study, which might have been related to the low number of surgeries. The arteries and veins have several distinctions which can be used for identification. (I) The veins are not full in appearance and are dark red in color; in contrast, most of arteries are bright red in color and full in appearance. (II) Arteries pulsate while veins do not; notably, the pulsation of some arteries may become less obvious after mechanical stimulation is applied and vasospasm is experienced. (III) The veins are larger in number and more complex, with an average of 11-13 veins on one side; in contrast, the arteries are much smaller in number, with an average of $1-3$ arteries on one side $(8,31)$. (IV) Many of the pampiniform veins have their branches, while most of the arteries do not have branches. Finally, (V) dripping of lidocaine or opium can stimulate the pulsation of the arteries, but veins no pulsation, so it is easy for surgeon to differentiate them. In addition, intraoperative vascular Doppler ultrasound can also be used for vascular identification $(8,32)$. Vas deferens injury is another serious complication of VC surgery. Wang et al. performed VC surgery in 3,565 cases, and vas deferens injury was reported in only 1 case (33). No vas deferens injury occurred in our study. During the surgery, we divided the spermatic cord into the cremasteric system, internal spermatic vein system, and vas deferens system based on the anatomical structures. The division of these three systems helped to standardize the surgical procedure and reduce the tissues exposed in the operative field, which could prevent missing or incorrect ligation of the internal spermatic vein and simultaneously protect the vas deferens. This method greatly improves the safety of the procedure, reduces the difficulty of the operation, and shortens the operative time. 
MSV with ERAS has many advantages. First, it significantly shortens the length of hospital stay and saves considerable time. It is also able to avoid general anesthesia or combined spinal-epidural anesthesia and can reduce the impact of anesthesia on body homeostasis. Moreover, no fasting or intravenous nutrition is needed and patients can eat normally, which further strengthens the body homeostasis. Additionally, patients are awake intraoperatively, which facilitates doctor-patient communication and increases the doctor-patient trust. Furthermore, because of the reductions in anesthesia time, awakening time, postoperative observation time, and postoperative nutritional rehydration time, the patient's turnaround is accelerated (shortened from weeks and days to hours); as a result, the medical costs are dramatically reduced by about $30-50 \%$. Moreover, local anesthesia allows further observation of the nerve courses, avoids nerve injury and thus prevents further adverse medical events such as postoperative intractable pain. Finally, due to the significant reduction in surgical time, the use of interventional materials (such as urinary catheters, gastric tubes, and drainage tubes) is dramatically decreased, which significantly lowers the risk of intraoperative and postoperative infections and effectively prevents the emergence of iatrogenic drug resistance.

\section{Conclusions}

MSV with ERAS is safe and feasible. After MSV, the semen quality improvement rate was over $80 \%$, the spontaneous conception rate was about $27 \%$, and the testicular pain relief rate was about $95 \%$. MSV under local anesthesia allows accurate separation of the internal spermatic vein system, preservation of arteries and lymphatic vessels, and skeletonization of the internal spermatic veins, which are keys to the success of the procedure and the reduction of postoperative complications. MSV may also help to improve the conception outcomes in patients with $\mathrm{VC}$ accompanied with sperm DNA fragmentation or nonobstructive azoospermia, but this assertion should be confirmed by further research.

\section{Acknowledgments}

We are grateful to all the medical and nursing staff at the Center for Reproductive Medicine, Shengjing Hospital of China Medical University for their active cooperation and efforts in this study. We thank them for their dedicated care and treatment of the patients.

Funding: This research is supported by the Grant for New Clinical Initiative 2020 of China Medical University.

\section{Footnote}

Reporting Checklist: The authors have completed the STROBE reporting checklist. Available at https://dx.doi. org/10.21037/tau-21-908

Data Sharing Statement: Available at https://dx.doi. org/10.21037/tau-21-908

Conflicts of Interest: All authors have completed the ICMJE uniform disclosure form (available at https://dx.doi. org/10.21037/tau-21-908). The authors have no conflicts of interest to declare.

Ethical Statement: The authors are accountable for all aspects of the work in ensuring that questions related to the accuracy or integrity of any part of the work are appropriately investigated and resolved. The study was approved by Ethics Committee of Shengjing Hospital of China Medical University (No. 2020PS083J) and written informed consent was obtained from all patients. The study was conducted in accordance with the Declaration of Helsinki (as revised in 2013).

Open Access Statement: This is an Open Access article distributed in accordance with the Creative Commons Attribution-NonCommercial-NoDerivs 4.0 International License (CC BY-NC-ND 4.0), which permits the noncommercial replication and distribution of the article with the strict proviso that no changes or edits are made and the original work is properly cited (including links to both the formal publication through the relevant DOI and the license). See: https://creativecommons.org/licenses/by-nc-nd/4.0/.

\section{References}

1. Barratt CLR, Björndahl L, De Jonge CJ, et al. The diagnosis of male infertility: an analysis of the evidence to support the development of global WHO guidancechallenges and future research opportunities. Hum Reprod Update 2017;23:660-80.

2. Boivin J, Bunting L, Collins JA, et al. International estimates of infertility prevalence and treatment-seeking: potential need and demand for infertility medical care. 
Hum Reprod 2007;22:1506-12.

3. Cho CL, Esteves SC, Agarwal A. Indications and outcomes of varicocele repair. Panminerva Med 2019;61:152-63.

4. Al-Kandari AM, Shabaan H, Ibrahim HM, et al. Comparison of outcomes of different varicocelectomy techniques: open inguinal, laparoscopic, and subinguinal microscopic varicocelectomy: a randomized clinical trial. Urology 2007;69:417-20.

5. Ding H, Tian J, Du W, et al. Open non-microsurgical, laparoscopic or open microsurgical varicocelectomy for male infertility: a meta-analysis of randomized controlled trials. BJU Int 2012;110:1536-42.

6. Al-Kandari AM, Khudair A, Arafa A, et al. Microscopic subinguinal varicocelectomy in 100 consecutive cases: Spermatic cord vascular anatomy, recurrence and hydrocele outcome analysis. Arab J Urol 2018;16:181-7.

7. Hopps CV, Lemer ML, Schlegel PN, et al. Intraoperative varicocele anatomy: a microscopic study of the inguinal versus subinguinal approach. J Urol 2003;170:2366-70.

8. Shiraishi K, Oka S, Matsuyama H. Surgical comparison of subinguinal and high inguinal microsurgical varicocelectomy for adolescent varicocele. Int J Urol 2016;23:338-42.

9. Jensen CFS, Østergren P, Dupree JM, et al. Varicocele and male infertility. Nat Rev Urol 2017;14:523-33.

10. Yuan R, Zhuo H, Cao D, et al. Efficacy and safety of varicocelectomies: A meta-analysis. Syst Biol Reprod Med 2017;63:120-9.

11. Cayan S, Acar D, Ulger S, et al. Adolescent varicocele repair: long-term results and comparison of surgical techniques according to optical magnification use in 100 cases at a single university hospital. J Urol 2005;174:20036; discussion 2006-7.

12. Ghanem H, Anis T, El-Nashar A, et al. Subinguinal microvaricocelectomy versus retroperitoneal varicocelectomy: comparative study of complications and surgical outcome. Urology 2004;64:1005-9.

13. Kim KH, Lee JY, Kang DH, et al. Impact of surgical varicocele repair on pregnancy rate in subfertile men with clinical varicocele and impaired semen quality: a metaanalysis of randomized clinical trials. Korean J Urol 2013;54:703-9.

14. Vermeulen A, Vandeweghe M, Deslypere JP. Prognosis of subfertility in men with corrected or uncorrected varicocele. J Androl 1986;7:147-55.

15. Lund L, Larsen SB. A follow-up study of semen quality and fertility in men with varicocele testis and in control subjects. Br J Urol 1998;82:682-6.
16. Peterson AC, Lance RS, Ruiz HE. Outcomes of varicocele ligation done for pain. J Urol 1998;159:1565-7.

17. Paick S, Choi WS. Varicocele and Testicular Pain: A Review. World J Mens Health 2019;37:4-11.

18. Al-Gadheeb A, El-Tholoth HS, Albalawi A, et al. Microscopic subinguinal varicocelectomy for testicular pain: a retrospective study on outcomes and predictors of pain relief. Basic Clin Androl 2021;31:1.

19. Han DY, Yang QY, Chen X, et al. Who will benefit from surgical repair for painful varicocele: a meta-analysis. Int Urol Nephrol 2016;48:1071-8.

20. Majholm B, Engbæk J, Bartholdy J, et al. Is day surgery safe? A Danish multicentre study of morbidity after 57,709 day surgery procedures. Acta Anaesthesiol Scand 2012;56:323-31.

21. Alacadag M, Cilingir D. Presurgery Anxiety and Day Surgery Patients' Need for Information. J Perianesth Nurs 2018;33:658-68.

22. Walker KJ, Smith AF. Premedication for anxiety in adult day surgery. Cochrane Database Syst Rev 2009;(4):CD002192.

23. Zhang MS, Sun PY, Liu MZ, et al. A case report of a woman after childbirth with a dehisced abdominal wound as well as fat liquefaction and large skin necrosis. Ann Palliat Med 2020;9:493-6.

24. Szabo R, Kessler R. Hydrocele following internal spermatic vein ligation: a retrospective study and review of the literature. J Urol 1984;132:924-5.

25. Guo L, Sun W, Shao G, et al. Outcomes of Microscopic Subinguinal Varicocelectomy With and Without the Assistance of Doppler Ultrasound: A Randomized Clinical Trial. Urology 2015;86:922-8.

26. Zini A, Fischer A, Bellack D, et al. Technical modification of microsurgical varicocelectomy can reduce operating time. Urology 2006;67:803-6.

27. Song Y, Lu Y, Xu Y, et al. Comparison between microsurgical varicocelectomy with and without testicular delivery for treatment of varicocele: A systematic review and meta-analysis. Andrologia 2019;51:e13363.

28. Ramasamy R, Schlegel PN. Microsurgical inguinal varicocelectomy with and without testicular delivery. Urology 2006;68:1323-6.

29. Rotker K, Sigman M. Recurrent varicocele. Asian J Androl 2016;18:229-33.

30. Chan PT, Wright EJ, Goldstein M. Incidence and postoperative outcomes of accidental ligation of the testicular artery during microsurgical varicocelectomy. J Urol 2005;173:482-4. 
31. Lv KL, Zhuang JT, Zhao L, et al. Varicocele anatomy during subinguinal microsurgical varicocelectomy in Chinese men. Andrologia 2015;47:1190-5.

32. Cocuzza M, Pagani R, Coelho R, et al. The systematic use of intraoperative vascular Doppler ultrasound during microsurgical subinguinal varicocelectomy improves precise identification and preservation of testicular blood supply. Fertil Steril 2010;93:2396-9.

33. Wang J, Liu Q, Wang X, et al. Modified Inguinal Microscope-Assisted Varicocelectomy under Local Anesthesia: A Non-randomised Controlled Study of 3565 Cases. Sci Rep 2018;8:2800.

(English Language Editor: J. Gray)
Cite this article as: Wang X, Wang R, Du Q, Pan B. Clinical effectiveness of microsurgical subinguinal varicocelectomy with enhanced recovery after surgery for varicocele. Transl Androl Urol 2021;10(10):3862-3872. doi: 10.21037/tau-21-908 